\title{
Dynamic QoE-aware live streaming with clustering clients
}

\author{
Naoki Satake ${ }^{a}$ and Masaki Bandai \\ Dept. of Information and Communication Sciences, Sophia University \\ 7-1 Kioicho, Chiyda, Tokyo 102-8554, Japan \\ a)naoki.satake@sophia.ac.jp
}

\begin{abstract}
In this paper, we propose a dynamic Quality of Experience (QoE)-aware live streaming algorithm by utilizing client clustering and similarity metrics. Encoding scheme of the proposed system finds a sufficient bitrate of the video and creates quality levels adapted to the distribution of clients' bandwidth to improve overall average of user's QoE. We show that the proposed system can improve structural similarity method (SSIM) performance when the client's bandwidth is distributed in low or middle bandwidth, and decreases the number of clients who stop play back.
\end{abstract}

Keywords: video streaming, video quality of experience

Classification: Multimedia Systems for Communications

\section{References}

[1] L. D. Cicco, S. Mascolo, and V. Palmisano, "Feedback Control for Adaptive Live Streaming," ACM MMSys'11, pp. 145-156, Feb. 2011.

[2] Z. Wang, A. C. Bovic, H. R. Sheikh, and E. P. Simoncelli, "Image Quality Assessment: From Error Visibility to Structural Similarity," IEEE Trans. Image Process., vol. 13, no. 4, April 2004.

[3] T. Zinner, O. Abboud, O. Hohlfeld, and T. Hossfeld, "Impact of Frate Rate and Resolution on Objective QoE Metrics," QoMEX' 2010, pp. 2934, June 2010.

[4] T. Kanungo, D. M. Mount, N. S. Netanyahu, C. D. Piatko, R. Silverman, and A. Y. Wu, "An Efficient k-Means Clustering Algorithm: Analysis and Implementation," IEEE Trans. Pattern Anal. Mach. Intell., vol. 24, no. 7, July 2002 .

[5] H. Riiser, P. Vigmostad, C. Griwodz, and P. Halvorsen, "Bitrate and video quality planning for mobile streaming scenarios using a GPS-based bandwidth lookup service," IEEE ICME'11, pp. 11-15, July 2011.

[6] "Elephant's Dream," [Online] http://orange.blender.org

\section{Introduction}

Nowadays, live streaming is widely used in multiple ways: surveillance system, personal video and sports broadcasting. Scalable and adaptive video 
rate encoding scheme to realize smooth play back in various network conditions is necessary. Stream-switching is one of the most representative adaptation techniques, and is used for some streaming services. Server encodes video at several different bitrates, and informs clients the number of quality levels and their bitrates. Streaming-switching is mainly used for on-demand streaming, and has a problem to be adapted for live streaming. [1] presents an effective quality adaptation controller for a stream-switching video streaming system by using feedback control theory. However, it does not focus on the following problems. First problem is that the quality level is fixed in the system. If a server encodes the video to be adapted to each client's bandwidth completely, we can obtain high quality of experience (QoE) easily. However, it is difficult to realize due to the resource limitation of servers. Then, we can get higher QoE by adapting quality levels of encoding bitrates to the distribution of client's bandwidth. Second one is that a sufficient bitrate of the live-streaming content is unknown beforehand. If a server can know a sufficient bitrate of the content, clients obtain higher QoE by subdividing quality level within the limited bitrate.

In this paper, we propose a dynamic QoE-aware live streaming algorithm by utilizing client clustering method and similarity metrics, which can improve overall average of user's QoE by providing an efficient bitrate.

\section{Proposed System}

The proposed system aims to create dynamic quality levels. The proposed system renews the quality levels periodically. The renewal interval of setting quality level is defined as $T$ seconds. The proposed system composes of three steps: evaluating similarity step and clustering clients step, and creating quality levels.

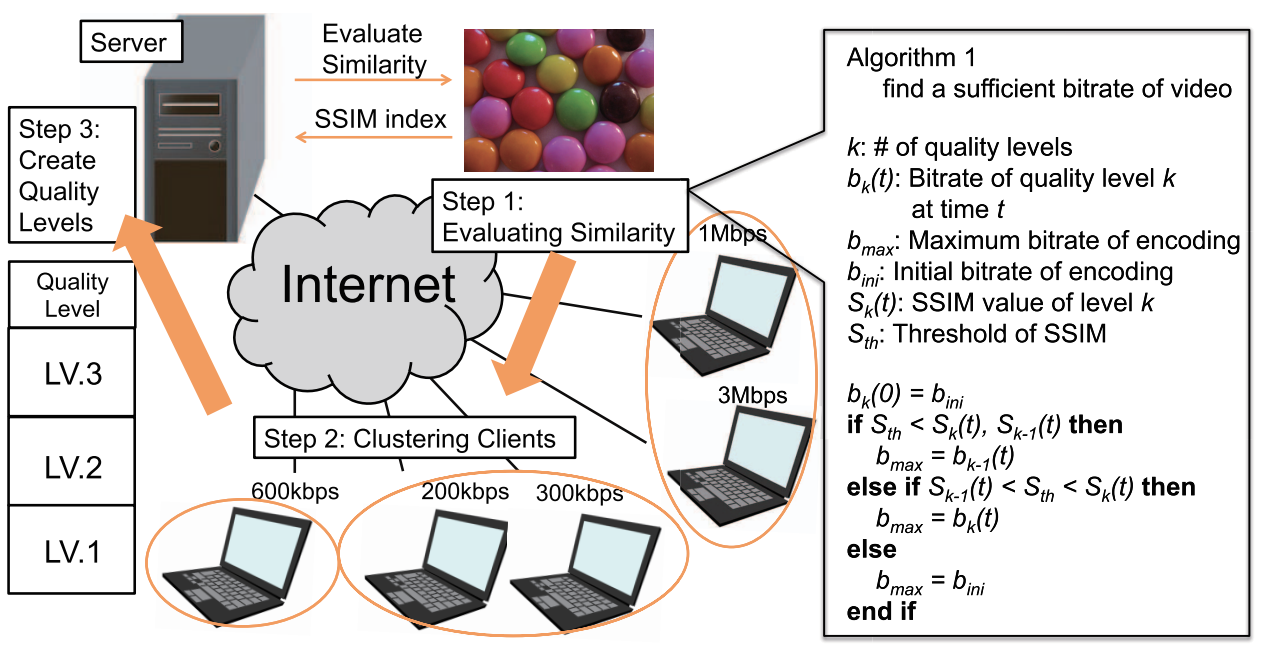

Fig. 1. System Architecture.

Fig. 1 illustrates the architecture of the proposed system. Step 1 shows evaluating similarity step to limit encoding bitrate. This process compares the similarity of a received image and its original one, and finds a sufficient 
bitrate of video. Step 2 shows the clustering step. This process groups clients into $k$ groups according to available bandwidth. The server calculates average of bitrate in each group. Finally, in Step 3, the server makes new quality levels according to the result of the previous two steps. The delay to obtain quality levels via Steps 1 to 3 is ignorable since calculating time is much smaller than $T$. After creating the quality levels, the server informs them to all clients. Each client requests for the server one of the bitrates.

\subsection{Evaluating Similarity (Step 1)}

As Step 1, evaluating similarity is necessary to set a maximum encoding bitrate to know the sufficient bitrate of the content and subdivide quality levels in the next step. Since the sufficient bitrate is different in video contents to get high QoE, we should decide encoding bitrate depending on the content type. The proposed system uses SSIM index [2] to limit bitrate of encoding.

Algorithm 1 in Fig. 1 shows how to find a sufficient bitrate of the video by using SSIM index. A server calculates SSIM of images encoded in the highest level $S_{k}(t)$ and the second highest level $S_{k-1}(t)$. Then, the server compares the SSIM indexes. The proposed algorithm considers only top two levels because of algorithmic simplicity. [3] indicates that human cannot recognize the difference between the encoded images and the original images when SSIM index is greater than 0.99. We regard that the quality of encoded image and the quality of original image are same when SSIM index of the image reaches 0.99 . Therefore, in this work, we set $S_{t h}=0.99$. We compare $S_{k-1}, S_{k}$ and $S_{t h}$. When SSIM indexes at level $k$ and $k-1$ are both higher than $S_{t h}$, we can decrease the maximum encoding bitrate $b_{\max }=b_{k-1}(t)$. If SSIM index at level $k-1$ is insufficient, we set $b_{\max }=b_{k}(t)$. When both SSIM indexes are lower than $S_{t h}, b_{\max }$ returns to $S_{i n i}$. In addition, $b_{i}$ $(i=0,1, \ldots, k)$ are given in Step 3 .

\subsection{Clustering Clients (Step 2)}

Step 2 creates the dynamic quality level by clustering clients. We use $k$ means [4] method for clustering clients. We represent client's bandwidth in time duration $[t-T, t]$ as $B(t)$, and a standard deviation in bandwidth as $\sigma(B(t))$ for the client. $n$ is the number of clients. In general, a smaller bitrate should be selected when standard deviation is larger considering bandwidth stability. Therefore, as same in [5], we use standard deviation in addition to average bandwidth. A server classifies clients into $k$ groups, and renews the bitrate of all quality levels according to the average bandwidth of each client in the same group. $k$ is the number of quality levels. Step 2 is divided into the following four Substeps:

1) A server receives $x_{j}$ from all clients. $x_{j}, j \in\{1, \ldots, n\}$ is defined as $x_{j}=\left(B_{j}(t), \sigma\left(B_{j}(t)\right)\right)$. To avoid encoding over $b_{\max }$, change $B(t)$ of the elements whose bandwidth is greater than maximum bitrate to $b_{\max }$. 
2) The server selects $k$ clients from all clients randomly, and gives a number $i \in\{1, \ldots, k\}$ to each client. $c_{i}$ is the center of each cluster $i$ and is defined as $c_{i}=\left(B_{i}(t), \sigma\left(B_{i}(t)\right)\right)$. The use of k-means in the proposed system is to find the minimum $\phi$.

$$
\phi=\sum_{x_{j} \in X} \min _{i \in k}\left\|x_{j}-c_{i}\right\|^{2}
$$

The server calculates Euclid distance between all $x_{j}$ and each $c_{i}$. The server assigns $x_{j}$ to the closest cluster $i . X$ is a set containing all $x_{j}$ s.

3) The server updates $c_{i}$ of each group by

$$
c_{i}=\frac{1}{\left|C_{i}\right|} \sum_{x_{j} \in C_{i}} x_{j}
$$

Here, $C_{i}$ is defined as the data set in the cluster $i$. In addition, $\left|C_{i}\right|$ is the number of data in the cluster $i$.

4) The server continues clustering while $c_{i}$ is changed from the previous result. Once the result is unchanged by the previous result, the server finishes the clustering.

\subsection{Creating Quality Levels (Step 3)}

In Step 3, the server decides the encoding bitrate using clustering result by

$$
b_{i}=B_{i}(t)+\alpha \cdot \sigma\left(B_{i}(t)\right)
$$

where $\alpha$ is a weight of $\sigma\left(B_{i}(t)\right)$. The server updates cluster number $i$ based on the ascending-order of $b_{i}$. For example, we assume $b_{1}=2 \mathrm{Mbps}, b_{2}=1 \mathrm{Mbps}$, and $b_{3}=3 \mathrm{Mbps}$ before cluster-number update. In that case, these cluster numbers are updated to $b_{1}=1 \mathrm{Mbps}, b_{2}=2 \mathrm{Mbps}$, and $b_{3}=3 \mathrm{Mbps}$. Therefore, cluster $k$ has the greatest bandwidth after updating cluster number. The initial values of $b_{i}$ are given as system parameters.

\section{Performance Evaluation}

In this section we evaluate the proposed system. We compare SSIM indexes between the videos being encoded at fixed encoding levels (conventional) and those being encoded in dynamic encoding levels (proposed). We use the video sequence "Elephant's Dream" [6] and encode it by FFmpeg library with x264 codecs. We set the number of encoding levels $k$ is 5 and encode videos in 240 seconds duration, and $30 \mathrm{fps}, 30 \mathrm{GOPs}, 1,280 \times 720$ resolution and five different bitrates. The bitrates of each 5 level are $\{300,700,1,500,2,500$, $3,500\}$, we use them as static levels of fixed encoding and default bitrate for the proposed method. We set the number of clients equals to 20 and 100. The interval of the new quality level creation $T$ is set to 30 seconds. In order to evaluate which condition the proposed system is effective, we prepare the following three types of client dataset:

1) Client's bandwidth is Gaussian distribution with $\mu=2 \mathrm{Mbps}$ and $\sigma=$ 400 
2) Client's bandwidth is Gaussian distribution with $\mu=500 \mathrm{kbps}$ and $\sigma=100$

3) Client's bandwidth is uniformly distributed between $10 \mathrm{kbps}$ to $4 \mathrm{Mbps}$ For all conditions, we define the probability density function by a standard deviation. We set $\mu$ equals to the bandwidth $B(t)$ of each client, and $\sigma$ equals to $1 / 5$ value of $B(t)$.

The result of received SSIM indexes is shown in Fig. 2. SSIM is the average of thirty seconds. In the result of Dataset 1, we observe that the proposed dynamic quality levels keep higher SSIM than the conventional fixed quality levels. SSIM index of the dynamic quality levels is higher than the fixed encoding level by 0.005 at time $=150$ in 100 clients. From 60 to 90 seconds and 150 to 180 seconds, 210 to 240 seconds, there is almost no difference between two systems. This is because around $1,500 \mathrm{kbps}$ is a sufficient bitrate to get enough quality. On the other hand, bitrate limitation occurs at time $=60,210$. At time $=60$, the server limits to $2,500 \mathrm{kbps}$. Since $2,000 \mathrm{kbps}$ is a sufficient bitrate, we can get good result by subdividing quality levels under 2,500 kbps. From the result of Dataset 2, the dynamic quality levels keeps higher SSIM index than the fixed quality levels.

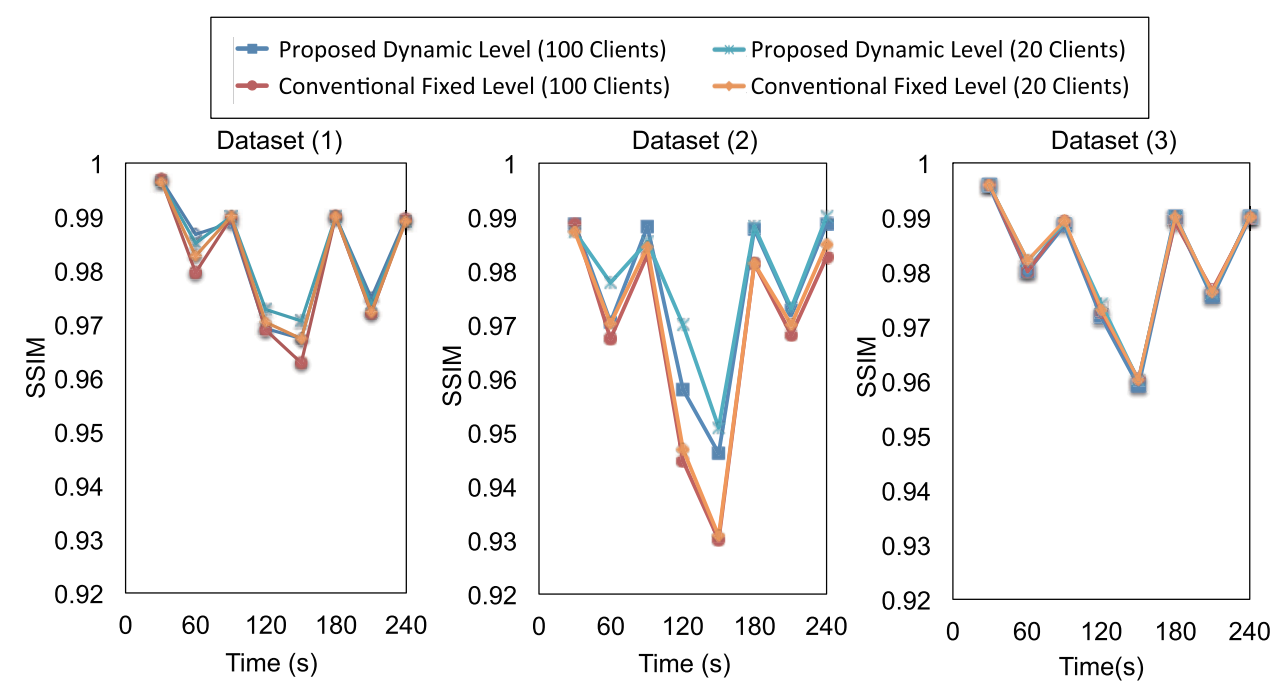

Fig. 2. SSIM performance.

At time $=150$ in 100 clients, SSIM index improves nearly 0.016. As well as Dataset 1, we can obtain the SSIM performance improvement in Dataset 2 because of the same reason mentioned in Dataset 1. From the result of Dataset 3, SSIM indexes of both systems are almost the same. Since we generate clients' bandwidths in Uniform distribution from $10 \mathrm{kbps}$ to $4 \mathrm{Mbps}$, there is no benefit to use clustering.

The result of the number of clients who stop play back is shown in Fig. 3. We can observe that the number of clients who stop play back is decreased in both Dataset 2 and 3. In both Dataset 2 and 3, some clients' bandwidths become under $300 \mathrm{kbps}$. In these clients, the video stops play back in the conventional fixed quality levels. Since the proposed dynamic quality levels 


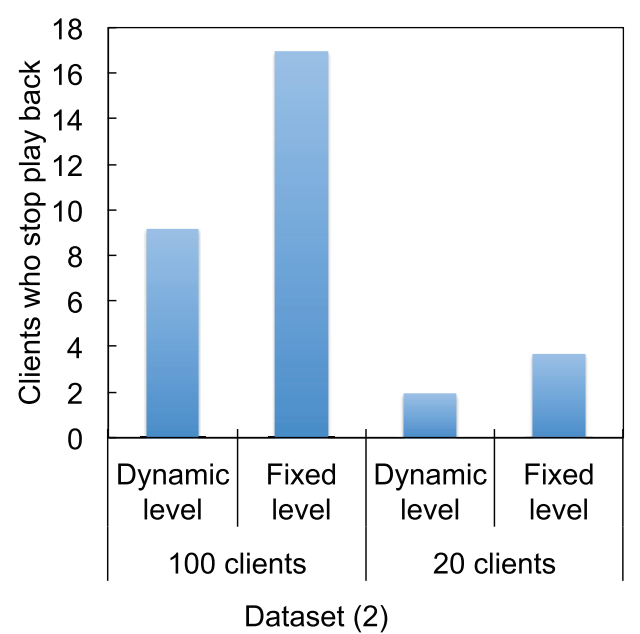

Fig. 3. Number of clients who stop play back in Dataset 2 and 3.

can create quality levels under $300 \mathrm{kbps}$, the number of clients who stop play back is decreased compared to the conventional fixed quality levels. We do not show the result of Dataset 1 because none of clients stop play back. We can confirm that the proposed method is effective to decrease the number of clients who stop play back of the proposed method in all cases.

\section{Conclusion}

In this paper, we have proposed a dynamic QoE-adaptive live streaming with clustering clients. The proposed system creates quality levels adapted to the distribution of client's bandwidth by using clustering method and evaluating similarity of encoded images. We have evaluated the proposed system from the perspective of SSIM index, and confirmed that the proposed method can improve user's QoE performance (SSIM index) in the condition that the distribution of client's bandwidth is concentrated in some range, and decrease the number of clients who stop play back. In future, we modify the proposed method to consider more than top three levels of SSIMs. In addition, we evaluate delay performance of the proposed system quantitatively. 